\title{
ОДНОВИБРАТОРЫ НА ЛОГИЧЕСКИХ ИНВЕРТОРАХ ТТЛ
}

\author{
(Представлена Н. Алумяэ)
}

\begin{abstract}
Проанализированы основные типы одновибраторов на логических ннверторах транзисторно-транзисторной логики (ТТЛ). Определены их параметры и приведены результаты экспериментального исследования. Показано, что наилучшими характеристиками в наносекундном диапазоне обладает схема, содержащая логический инвертор, между входом и выходом которого подключен времязадающий конденсатор и на входе которого находится ключ, выполненный на основе логического инвертора с открытым коллекторным выходом.
\end{abstract}

Одновибраторы (ждущие мультивибраторы) являются одними из основных функциональных элементов любого цифрового устройства. Они используются для формирования и селекции импульсов, для реализации схем задержки и времязадающих цепей и т. д. В ТТЛ-схемах одновибраторы могут быть выполнены либо на элементах специального типа, либо на основе простейших логических инверторов (в частности, на стандартных двухвходовых ячейках И-НЕ). Последний тип одновибраторов отличается простотой и экономичностью исполнения, а в наносекундном диапазоне также некоторыми преимуществами в технических параметрах. Это обусловлено тем, что специальные микросхемы (SN74121, K155AГ1 и им подобные) имеют сравнительно большое время внутренней задержки (минимально 50 нсек, максимально 80 нсек) и весьма длительное время восстановления при малых значениях хронирующего резистора (допустимый коэффициент заполнения импульсов может достигать $67 \%)$.

Известен целый ряд одновибраторов на логических инверторах ТТЛ $\left[{ }^{1,2}\right]$, но оценка отдельных схем и выбор наиболее подходящего варианта сильно затруднены, так как информация об их основных характеристиках либо отсутствует, либо она крайне недостаточна.

В настоящей статье представлены достаточно полные данные об основных параметрах таких одновибраторов и рассмотрены, кроме того, некоторые новые схемы с улучшенными характеристиками. Вне анализа оставлены все громоздкие с точки зрения практической реализации варианты, в том числе схемы с более чем двумя внешними элементами типа конденсатора, резистора или диода, а также схемы, содержащие дополнительные дискретные транзисторы, линии задержки и т. д. На этом же основании рассматриваются только одновибраторы с потенциальными цепями запуска, хотя в некоторых случаях возможно применение и дифференцирующих входных цепей (у т. н. удлинителей импульсов, для которых, как известно, входный запускающий импульс должен быть короче выходного). 


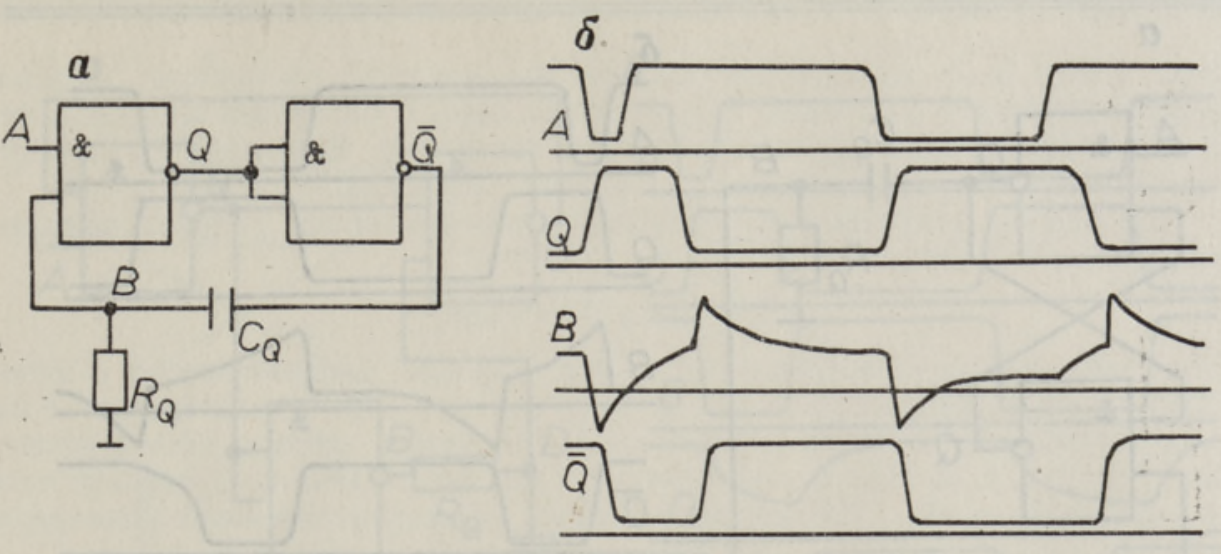

Рис. 1 .

На рис. 1, a показана схема одновибратора с двумя последовательно включенными инверторами с $R_{Q} C_{Q}$-цепочкой между выходом второго и входом первого инвертора. Его работа поясняется импульсной диаграммой на рис. 1,б. Величина резистора $R_{Q}$ определяется условием

$$
R_{Q}>R_{k r}=\left(U_{T} R_{b}\right) /\left(E-U_{b e}-U_{T}\right),
$$

где $U_{T}$ - пороговое напряжение переключения инвертора, $R_{b}-$ базовое сопротивление входного (многоэмиттерного) транзистора инвертора, $E$ - напряжение питания, $U_{b e}-$ падение напряжения на переходе база-эмиттер входного (многоэмиттерного) транзистора инвертора.

На основе решения дифференциального уравнения

$$
C_{Q} R_{e k v} \frac{d U_{B}}{d t}+U_{B}=E_{e k v},
$$

где $R_{e k v}=\left(R_{Q} R_{b}\right) /\left(R_{Q}+R_{b}\right), \quad E_{e k v}=\left[R_{Q}\left(E-U_{b e}\right)\right] /\left(R_{Q}+R_{b}\right)$, $U_{B}-$ напряжение в точке $B$, получим, что

$$
t_{Q}=C_{Q} R_{e h v} \ln \left[1+\left(U^{1}-U^{0}\right) /\left(E_{e h v}-U_{T}\right)\right],
$$

где $U^{1}-$ напряжение логической 1 и $U^{0}-$ напряжение логического 0 .

Этот одновибратор может работать только в режиме удлинителя импульсов, причем стабильность $t_{Q}$ при изменении напряжения питания и температуры невысокая и время восстановления большое. Известен ряд модификаций этой схемы: а) подключение $R_{Q}$ к источнику питания, б) подключение $C_{Q}$ к резистивному делителю напряжения, в) подключение $C_{Q}$ к делителю напряжения, одно плечо которого представляет собой диодную цепочку, что существенно сокращает время восстановления.

На рис. 2, а изображен известный тип одновибратора на логических инверторах с дифференцирующей $R_{Q} C_{Q}$-цепочкой и его работа иллюстрируется импульсной диаграммой на рис. 2, б. Этот одновибратор в принципе универсален (т. е. длительность запускающего импульса $t_{A}$ может быть как короче, так и длиннее $t_{Q}$ ), но в случае $t_{A}>t_{Q}$ импульс на выходе $\bar{Q}$ получается удлиненным за счет затянутости заднего фронта.

Длительность выходного импульса может быть рассчитана по формуле 


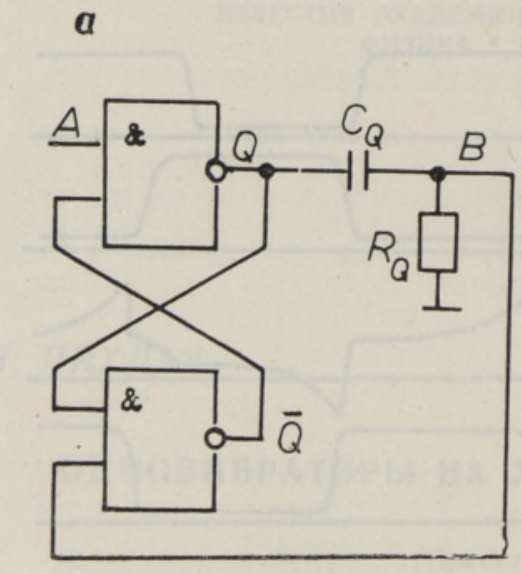

$\delta$

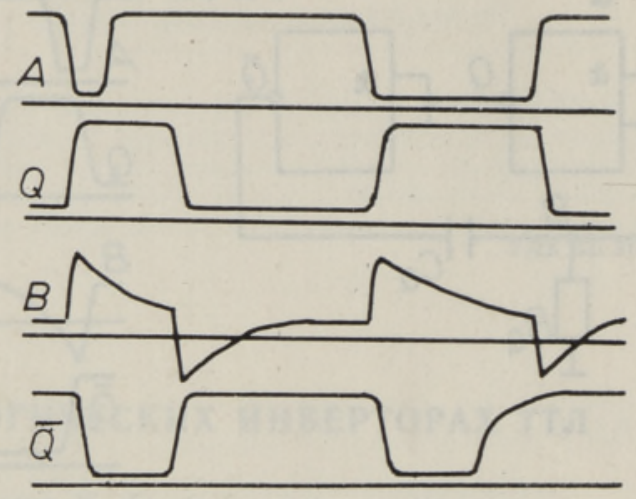

Рис. 2 .
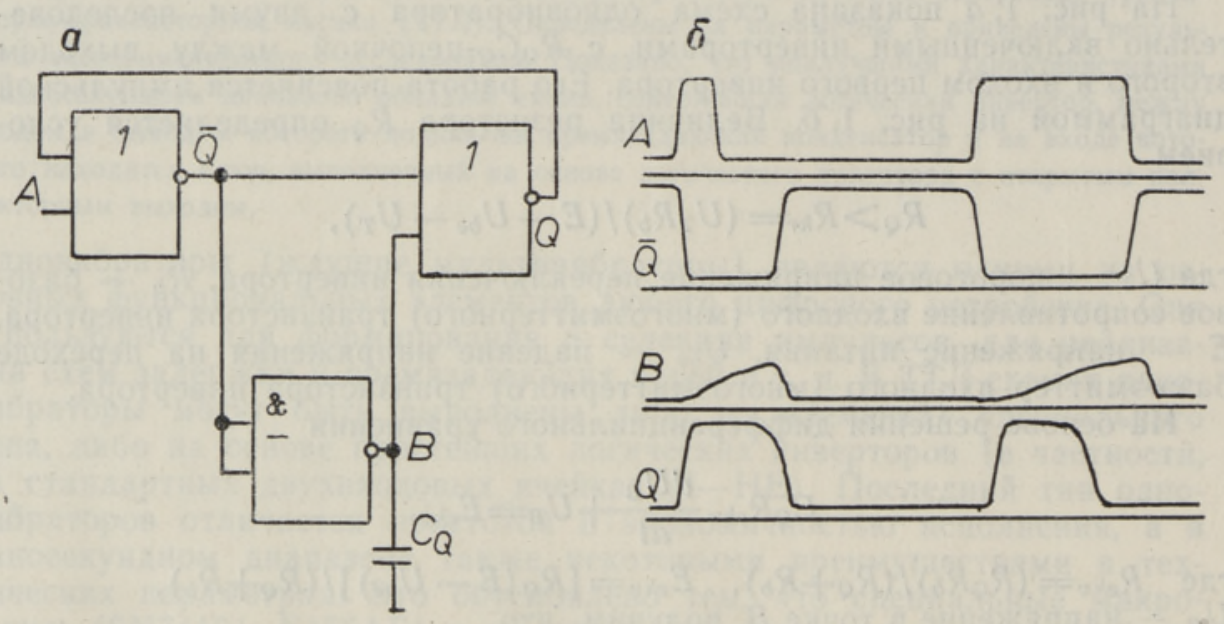

Pнс. 3 .

$$
t_{Q}=C_{Q}\left(R_{Q}+R_{V}\right) \ln \frac{U^{1}-U^{0}+\left[\left(E-U_{b e}\right) R_{Q}\right] /\left(R_{Q}+R_{b}\right)}{U_{T}} \frac{R_{Q}}{R_{Q}+R_{V}},
$$

где $R_{V}$ - выходное сопротивление инвертора в состоянии 1 .

Выбор величины $R_{Q}$ ограничен как сверху (в состоянии покоя $U_{B}$ должно быть ниже $U_{T}$ ), так и снизу (при запуске одновибратора скачок $U_{B}$ должен превышать $U_{T}$ ), поэтому обычно $R_{Q}$ выбирается в пределах 220-680 Ом. Для улучшения отдельных характеристик схемы 2, $a$ предложено немало способов, среди которых можно указать: a) включение диода (диодов) термокомпенсации между точкой соединения $C_{Q}, R_{Q}$ и входом второго инвертора, б) замена резистора $R_{Q}$ эмиттерным или истоковым повторителем.

Одновибратор на рис. $3, a$ выполнен на элементах ИЛИ-НЕ и запускается положительным перепадом входного напряжения (у других для этого подключается дополнительный инвертор). Другой особенностью схемы является наличие в ней логического инвертора с откры- 
$a$

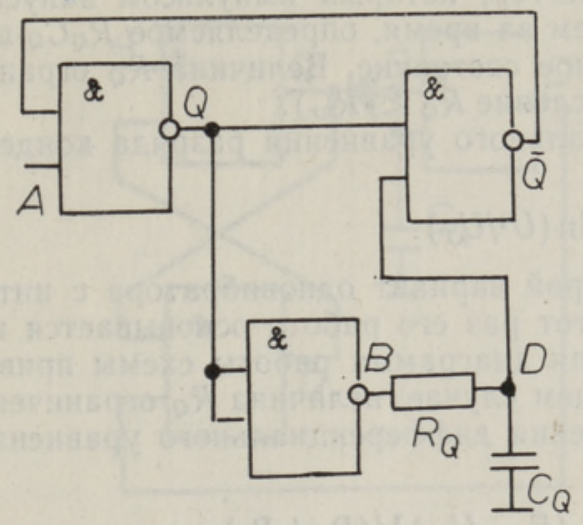

$\delta$

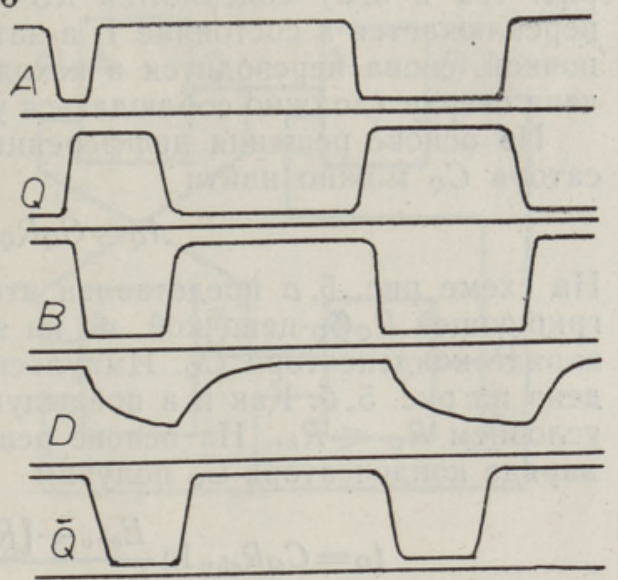

Рис. 4.
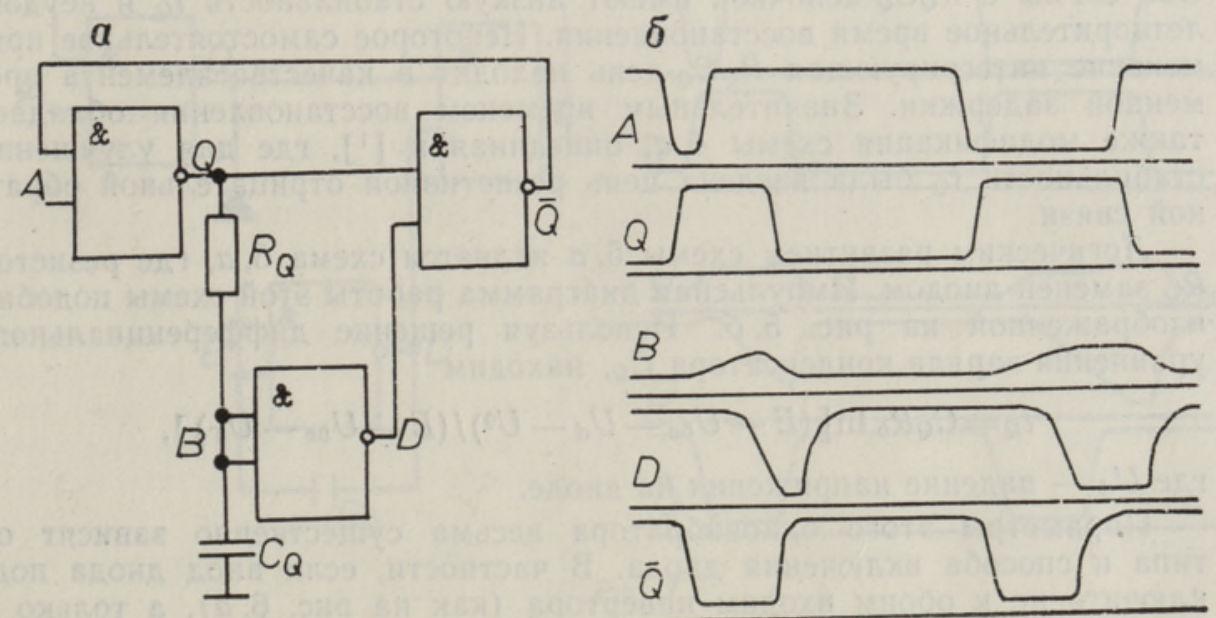

Рис. 5.

тым коллекторным выходом *, что обеспечивает малое время восстановления одновибратора. Импульсная диаграмма работы схемы приведена на рис. 3,6 . Заметим, что форма импульса на выходе $Q$ сильно искажена. Длительность квазистабильного состояния, во время которого формируется выходной импульс, определяется выражением

$$
t_{Q}=C_{Q} R_{b} \ln \left[\left(E-U_{b e}-U^{0}\right) /\left(E-U_{b e}-U_{T}\right)\right] .
$$

Работа одновибратора с интегрирующей $R_{Q} C_{Q}$-цепочкой на рис. $4, a$ основывается на разряде времязадающего конденсатора $C_{Q}$. Диаграмма импульсов представлена на рис. 4, б. Как и все последующие варианты, этот одновибратор универсален по выходу $\bar{Q}$. При этом нетрудно заметить, что в нем (как и в других, за исключением схем

* На рисунках инверторы с открытым коллекторным выходом отмечены черточкой под знаком логического снмвола. 
рис. $1, a$ и $3, a)$ содержится $R S$-триггер, который импульсом запуска переключается в состояние 1 , а затем за время, определяемое $R_{Q} C_{Q}$-цепочкой, снова переводится в исходное состояние. Величина $R_{Q}$ ограничена сверху (должно соблюдаться условие $R_{Q}<R_{k r}$ ).

На основе решения дифференциального уравнения разряда конденсатора $C_{Q}$ можно найти

$$
t_{Q}=C_{Q} R_{Q} \ln \left(U^{1} / U_{T}\right) .
$$

На схеме рис. $5, a$ представлен второй вариант одновибратора с интегрирующей $R_{Q} C_{Q}$-цепочкой, но на этот раз его работа основывается на заряде конденсатора $C_{Q}$. Импульсная диаграмма работы схемы приведена на рис. 5 , б. Как и в предыдущем случае, величина $R_{Q}$ ограничена условием $R_{Q}<R_{k r}$. На основе решения дифференциального уравнения заряда конденсатора $C_{Q}$ получим

$$
t_{Q}=C_{Q} R_{e k v} \ln \frac{E_{e h v}-\left[R_{Q}\left(E-U_{b e}\right)\right] /\left(R_{Q}+R_{b}\right)}{E_{e k v}-U_{T}},
$$

где $R_{e k v}=\left(R_{Q} R_{b}\right) /\left(R_{Q}^{\prime}+R_{b}\right), \quad E_{e k v}=R_{e k v}\left[U^{1} / R_{Q}+\left(E-U_{b e}\right) / R_{b}\right]$.

Обе схемы с $R_{Q} C_{Q}$-цепочкой имеют низкую стабильность $t_{Q}$ и неудовлетворительное время восстановления. Некоторое самостоятельное применение интегрирующая $R_{Q} C_{Q}$-цепь находит в качестве элемента временной задержки. Значительным временем восстановления обладает также модификация схемы $4, a$, описанная в [ $\left.{ }^{1}\right]$, где для улучшения стабильности $t_{Q}$ была введена цепь резистивной отрицательной обратной связи.

Логическим развитием схемы $5, a$ является схема $6, a$, где резистор $R_{Q}$ заменен диодом. Импульсная диаграмма работы этой схемы подобна изображенной на рис. 5,6 . Используя решение дифференциального уравнения заряда конденсатора $C_{Q}$, находим

$$
t_{Q}=C_{Q} R_{b} \ln \left[\left(E-U_{b e}-U_{d}-U^{0}\right) /\left(E-U_{b e}-U_{T}\right)\right],
$$

где $U_{d}$ - падение напряжения на диоде.

Параметры этого одновибратора весьма существенно зависят от типа и способа включения диода. В частности, если анод диода подключить не к обоим входам инвертора (как на рис. $6, a$ ), а только к одному, и второй вход инвертора соединить с катодом диода, то параметры одновибратора заметно ухудшаются. Интересно добавить, что в принципе можно обойтись вообще без диода, если один вход инвертора соединить непосредственно с точкой $Q$, а второй - с времязадающим конденсатором, который шунтируется резистором. Но характеристики этой схемы хуже, чем у варианта рис. $6, a$.

В одновибраторе на рис. $6, \sigma$ диод заменен ключом с применением логического инвертора с открытым коллекторным выходом. Импульсная диаграмма работы схемы сходна с изображенной на рис. $5, \sigma$ и выражение $t_{Q}$ аналогично полученному для схемы $3, a$.

Дальнейшего улучшения характеристик одновибраторов на логических инверторах можно добиться путем перевода работы инверторов в режим интегратора Миллера, когда между выходом и входом инвертора подключается интегрирующий конденсатор $\left[{ }^{3}\right]$. Это позволяет улучшать помехозащищенность триггерных и одновибраторных схем [4-6], а также создавать ряд интересных вариантов одновибраторов $\left[{ }^{7-9}\right]$.

Основной диодный вариант такого одновибратора показан на рис. $7, a$ и соответствующая импульсная диаграмма приведена на 

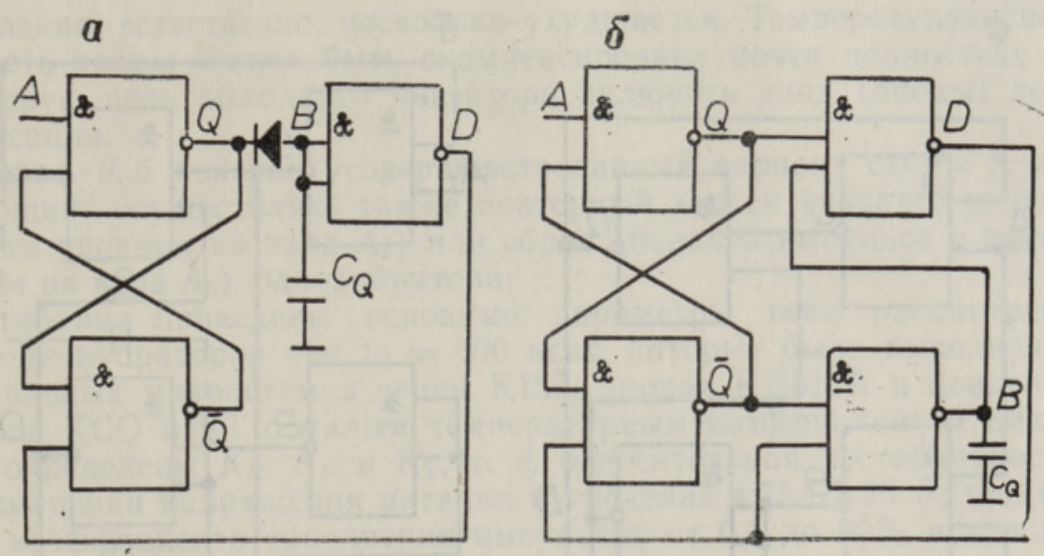

Рис. 6.

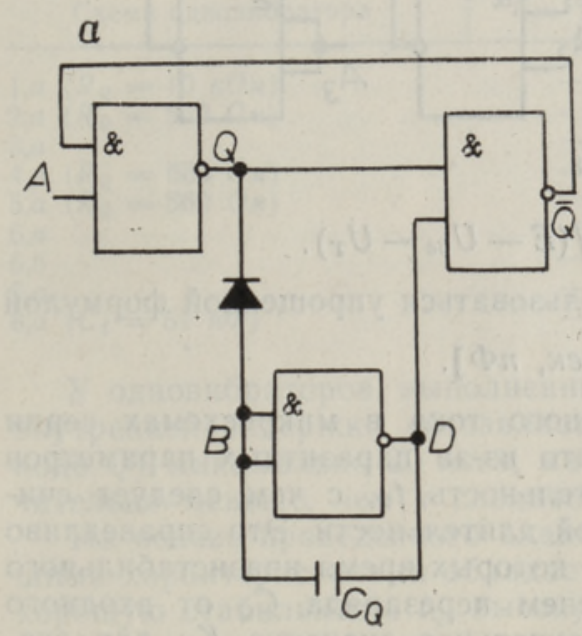

$\delta$
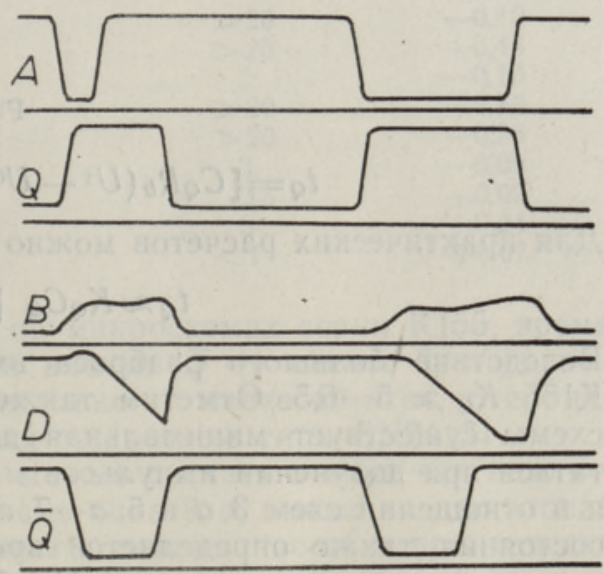

Рис. 7.

рис. 7, б. Учитывая, что во время формирования выходного импульса инвертор, имеющий времязадающий конденсатор между входом и выходом, работает как интегрирующий усилитель с практически постоянным током разряда $C_{Q}$, можно написать

$$
t_{Q}=\left[C_{Q} R_{b}\left(U^{1}-U_{d}-U^{0}\right)\right] /\left(E-U_{b e}-U_{T}\right) .
$$

Как и в случае варианта $6, a$, параметры этой схемы существенно зависят от типа и способа включения диода. Если анод диода подключен только к одному входу инвертора, а второй вход соединен с катодом диода, то характеристики схемы заметно ухудшаются $\left[{ }^{7-9}\right]$.

В одновибраторе на рис. $8, a$ вместо диода применен ключ с использованием логического инвертора с открытым коллекторным выходом. Импульсная диаграмма работы схемы подобна изображенной на рис. $7, \sigma$, но благодаря меньшему начальному уровню напряжения на времязадающем конденсаторе она обладает лучшей помехозащищенностью и несколько большей длительностью выходного импульса. Величина $t_{Q}$ определяется выражением 

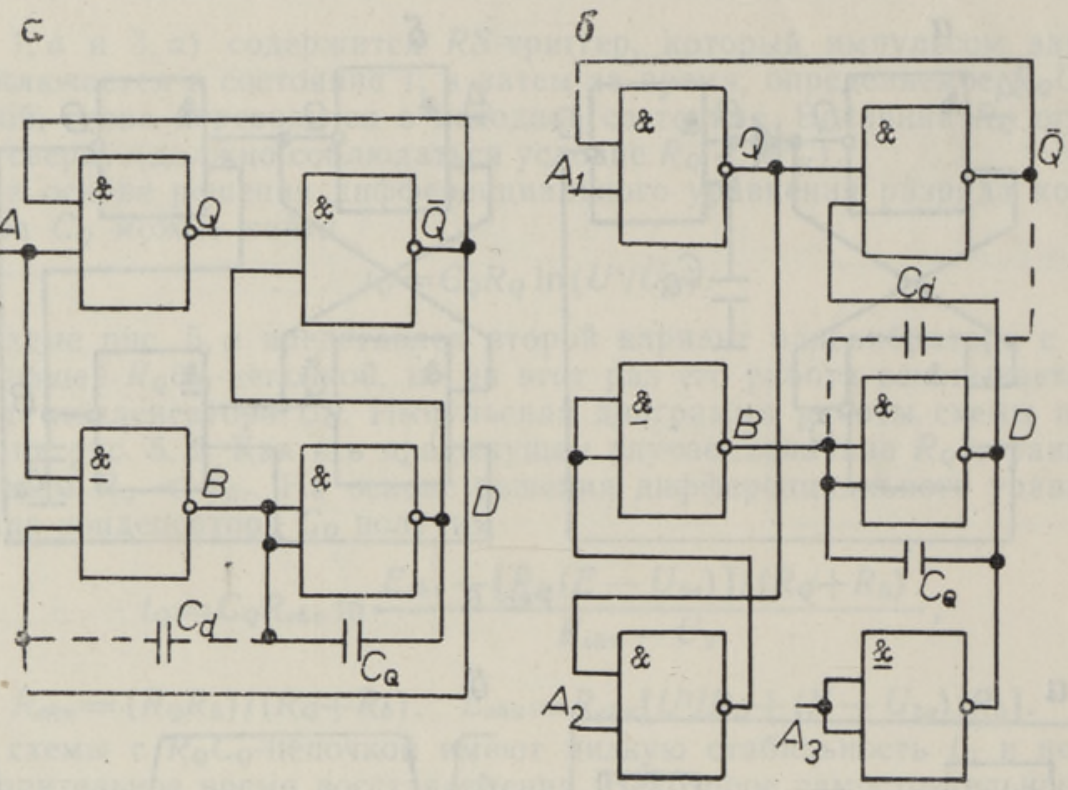

Рис. 8.

$$
t_{Q}=\left[C_{Q} R_{b}\left(U^{1}-U^{0}\right)\right] /\left(E-U_{b e}-U_{T}\right) .
$$

Для практических расчетов можно пользоваться упрощенной формулой

$$
t_{Q} \approx K_{Q} C_{Q}[н с е \kappa, n \Phi] .
$$

Вследствие большого разброса входного тока в микросхемах серии $\mathrm{K} 155 K_{Q} \approx 5-6,5$. Отметим также, что из-за паразитных параметров схемы существует минимальная длительность $t_{Q}$, с чем следует считаться при получении импульсов малой длительности. Это справедливо и в отношении схем $3, a$ и $5, a-7, a$, в которых время квазистабильного состояния также определяется временем перезаряда $C_{Q}$ от входного тока логического инвертора. Приблизительное значение $K_{Q}$ для схемы 6, б равно $1,5-2$, для схемы $7, a$ соответственно $4-5$.

Назначение конденсатора $C_{d}$ (показан на рис. $8, a$ и 6 пунктиром) требует особого разъяснения. Дело в том, что у всех вышеописанных одновибраторов используется нестандартный режим переключения инверторов медленно изменяющимся входным напряжением. Это приводит к затягиванию заднего фронта импульсов вплоть до самовозбуждения схемы. Предложенный в [ $\left.{ }^{9}\right]$ способ обратной связи через логические инверторы улучшает крутизну заднего фронта только частично, так как эффективное действие обратной связи начинается лишь с порогового уровня $U_{T}$. При включении же конденсатора $C_{d}$ крутизна заднего фронта резко повышается, а также обеспечивается стабильное переключение выходного инвертора. B наносекундном диапазоне $C_{d}$ может и отсутствовать, что оказывает некоторое влияние и на параметры одновибратора. Длительность $t_{Q}$ в широких пределах может быть отрегулирована потенциометром, который подключается параллельно времязадающему конденсатору $C_{Q}$.

Одновибратор на рис. 8, $a$ обладает сравнительно хорошей стабильностью $t_{Q}$ и ничтожно малым временем восстановления в наносекундном диапазоне. При больших значениях хронирующего конденсатора (т. е. при формировании импульсов большой длительности) время вос- 
становления, естественно, несколько ухудшается. Температурная нестабильность схемы может быть скомпенсирована почти полностью, если во входную цепь выходного инвертора включить диод (диоды) термокомпенсации.

На рис. 8,б показан усовершенствованный вариант схемы $8, a$, позволяющий осуществлять также повторный запуск (подачей импульса с низким уровнем на вход $A_{2}$ ) или сброс (подачей импульса с высоким уровнем на вход $A_{3}$ ) одновибратора.

В таблице приведены основные параметры всех рассмотренных здесь одновибраторов при $t_{Q}=500$ нсек, которые были выполнены на интегральных микросхемах серии K155, диодах КД512А и конденсатоpax типа КСО и КТ с малым температурным коэффициентом емкости. Были определены $K_{E}, K_{F}$ и $K_{T}$, т. е. относительная нестабильность $t_{Q}$ при изменении напряжения питания в пределах $4,75-5,25 B$, при изменении коэффициента заполнения импульсов от 0,1 до $90 \%$ и при изменении температуры в пределах $20-70^{\circ} \mathrm{C}$.

\begin{tabular}{l|c|c|c}
\hline Схема одновибратора & $K_{E}, \% / 0,1 B$ & $K_{F}, \%$ & $K_{T}, \% /{ }^{\circ} \mathrm{C}$ \\
\hline $1, a\left(R_{Q}=10 \kappa O M\right)$ & $-2,4$ & $>20$ & $-0,10$ \\
$2, a\left(R_{Q}=560 O M\right)$ & $+3,2$ & $>20$ & $+0,48$ \\
$3, a\left(R_{Q}=560 O M\right)$ & $-2,4$ & 2 & $-0,10$ \\
$4, a, a\left(R_{Q}=560 O M\right)$ & $+2,0$ & $>20$ & $+0,30$ \\
$6, a$ & $-2,4$ & 3 & $-0,26$ \\
6,6 & $-2,4$ & 2,5 & $-0,09$ \\
$7, a \quad\left(C_{d}=51 n \Phi\right)$ & $-0,4$ & 3 & $+0,24$ \\
$8, a\left(C_{d}\right.$ & $-1,0$ & $<1$ & $+0,07$
\end{tabular}

У одновибраторов, выполненных на микросхемах серии К 155 , время внутренней задержки составляет на выходе $\bar{Q}$ (у схемы $3, a$ на выходе $Q$ ) минимально 20 нсек, максимально - менее 40 нсек, т. е. значительно меньше, чем у специальных микросхем типа SN74121.

На основе проведенного анализа можно сделать вывод, что наилучшими характеристиками обладает схема типа $8, a$, имеющая достаточно хорошую стабильность $t_{Q}$, высокую поме́хозащищенность и практически нулевое время восстановления в наносекундном диапазоне.

\section{ЛИТЕРАТ У Р А}

1. Дьяконов В. П., Импульсные устройства на интегральных микросхемах, М., Изд. МЭИ, 1977.

2. P e č h o u č e k, M., Sdělovaci techn., 25, № 7, 265-268 (1977).

3. F a i m a n, M., Electronics, 44, № 16, 59 (1971).

4. S t e in k a m p, W., Elektronik, 21, № 5, $157-159$ (1972).

5. J e 1 i n e k, J., Sdëlovaci techn., 21, № 10, 375-376 (1973).

6. Greiner, G., Radio-Fernsehen-Elektronik, 23, № 15, 496-499 (1974).

7. Ба рб а сов В. М., Белов Е. Н., Мерч ански й В. Г., Соловьев А. К., Авт. свид. СССР № 415782, Бюлл. изобр., № 6 (1974).

8. Н а гайник А. И., Гуменюк В. П., Легостаев В. А., Авт. свид. СССР № 474922, Бюлл. изобр., № 23 (1975).

9. С емен н че в В. Н., Авт. свид. СССР № 636776, Бюлл. изобр., № 45 (1978).

Институт кибернетики

Академии наук Эстонской ССР
Поступила в редакцию 19/VI 1979 


\section{J. PIHLAU}

\section{TTL LOOGILISTEL INVERTORITEL POHINEVAD OOTEMULTIVIBRAATORID}

Artiklis on esitatud TTL loogilistest invertoritest koostatud ootemultivibraatorite skeemide analüüsi ja eksperimentaalse uurimise tulemused. On näidatud, et parimaid parameetreid nanosekund-diapasoonis omab skeem, mis sisaldab kaks järjestikku ühendatud invertorit, millest esimene on lahtise kollektorväljundiga ja teise sisendi ning väljundi vahele on lülitatud integreeriv kondensaator.

\section{J. PIHLAU}

\section{MONOSTABLES WITH TTL INVERTERS}

In this paper the results of the analysis of monostable multivibrators (one-shots) with TTL logic inverters (NAND and NOR gates) are being reported. A review and comparison of basic circuits including some new circuits are being presented. These new circuits use inverters as Miller integrators with an integrating capacitor between input and output of the gate. In order to speed up the recovery of the circuit, an additional inverter with an open-collector output is employed. Experimentally the main characteristics (recovery time and effect of temperature and supply voltage on pulse width) of all circuits mentioned were measured.

It is shown that the circuit comprising two series-connected inverters, the first one of an open-collector variety and the second one employed as a Miller integrator features the best nanosecond-range characteristics, namely a good pulse-width stability, a short internal delay, and a very high duty cycle. 